ISSN 1855-3966 (printed edn.), ISSN 1855-3974 (electronic edn.)

ARS MATHEMATICA CONTEMPORANEA 17 (2019) 581-589

https://doi.org/10.26493/1855-3974.1866.fd9

(Also available at http://amc-journal.eu)

\title{
Lobe, edge, and arc transitivity of graphs of connectivity 1
}

\author{
Jack E. Graver, Mark E. Watkins \\ Department of Mathematics, Syracuse University, Syracuse, NY
}

Received 27 November 2018, accepted 3 October 2019, published online 9 December 2019

\begin{abstract}
We give necessary and sufficient conditions for lobe-transitivity of locally finite and locally countable graphs whose connectivity equals 1 . We show further that, given any biconnected graph $\Lambda$ and a "code" assigned to each orbit of $\operatorname{Aut}(\Lambda)$, there exists a unique lobe-transitive graph $\Gamma$ of connectivity 1 whose lobes are copies of $\Lambda$ and is consistent with the given code at every vertex of $\Gamma$. These results lead to necessary and sufficient conditions for a graph of connectivity 1 to be edge-transitive and to be arc-transitive. Countable graphs of connectivity 1 the action of whose automorphism groups is, respectively, vertextransitive, primitive, regular, Cayley, and Frobenius had been previously characterized in the literature.
\end{abstract}

Keywords: Lobe, lobe-transitive, edge-transitive, orbit, connectivity.

Math. Subj. Class.: 05C25, 05C63, 05C38, 20827

\section{Introduction}

Throughout this article, $\Gamma$ denotes a connected simple graph. Consider the equivalence relation $\cong$ on the edge-set $E \Gamma$ of $\Gamma$ whereby $e_{1} \cong e_{2}$ whenever the edges $e_{1}$ and $e_{2}$ lie on a common cycle of $\Gamma$. A lobe is a subgraph of $\Gamma$ induced by an equivalence class with respect to $\cong$. Equivalently, a lobe is a subgraph that either consists of a cut-edge with its two incident vertices or is a maximal biconnected subgraph ${ }^{1}$. A vertex is a cut-vertex if it belongs to at least two different lobes. Connected graphs other than $K_{2}$ have connectivity 1 if and only if they have a cut-vertex. Clearly no finite vertex-transitive graph admits a cut-vertex.

\footnotetext{
E-mail addresses: jegraver@syr.edu (Jack E. Graver), mewatkin@syr.edu (Mark E. Watkins)

${ }^{1}$ The term "lobe" is due to O. Ore [6]. We eschew the term "block" for this purpose, as it leads to ambiguity when discussing imprimitivity.
} 
Graphs of connectivity 1 whose automorphism groups have certain given properties have been characterized. Those whose automorphism groups are, respectively, vertextransitive, primitive, and regular were characterized in [5]. In particular, primitive planar graphs of connectivity 1 were characterized in $[11]^{2}$. Cayley graphs of connectivity 1 were characterized in [9]. Graphs of connectivity 1 with Frobenius automorphism groups were characterized in [10]. In the present work, we complete this investigation; we characterize graphs of connectivity 1 whose automorphism groups act transitively on their set of lobes. As a consequence, we obtain characterizations of edge-transitive graphs and arc-transitive graphs of connectivity 1 .

The conditions for a graph of connectivity 1 to be lobe-transitive or to be vertextransitive are independent; such a graph may have either property or neither one or both. Such is not the case for edge- and arc-transitivity. In Section 3 we give necessary and sufficient conditions for a graph to be lobe-transitive. We further show that, given any biconnected graph $\Lambda$ and a "list" of orbit-multiplicities of copies of Aut $(\Lambda)$, one can construct a lobe-transitive graph of connectivity 1 all of whose lobes are isomorphic to $\Lambda$ and locally respects the given list. We give necessary and sufficient conditions for a countable graph of connectivity 1 to be edge-transitive in Section 4 and to be arc-transitive in Section 5. As the sets of conditions for these latter two properties are more intertwined with lobe-transitivity than the characterization of vertex-transitivity (for graphs of connectivity 1), scattered throughout are examples that illustrate some algebraic distinctions among these various properties.

\section{Preliminaries}

Throughout this article, the symbol $\mathbb{N}$ denotes the set of positive integers. The symbols $\mathbb{I}, \mathbb{J}$, and $\mathbb{K}$, often subscripted, denote subsets of $\mathbb{N}$ of the form $\{1,2, \ldots, n\}$ or the set $\mathbb{N}$ itself; they appear as sets of indices. All graphs (and their valences) in this article are finite or countably infinite. The symbol $\delta_{i, j}$ (the so-called "Kronecker delta") assumes the value 1 if $i=j$ and 0 if $i \neq j$. For a graph $\Lambda$ and any subgroup $H \leq \operatorname{Aut}(\Lambda)$, the set of orbits of $H$ acting on $V \Lambda$ is denoted by $\mathscr{O}(H)$.

The set of lobes of a graph $\Gamma$ is denoted by $\mathscr{L}(\Gamma)$. We let $\left\{\mathscr{L}_{k}: k \in \mathbb{K}\right\}$ denote the partition of $\mathscr{L}(\Gamma)$ into isomorphism classes of lobes. For given $k \in \mathbb{K}$ and a lobe $\Lambda \in \mathscr{L}_{k}$, we let $\mathscr{O}(\operatorname{Aut}(\Lambda))=\left\{(V \Lambda)_{j}: j \in \mathbb{J}_{k}\right\}$, and we understand that if $\sigma: \Lambda \rightarrow \Theta$ is an isomorphism between lobes in $\mathscr{L}_{k}$, then $\sigma\left((V \Lambda)_{j}\right)=(V \Theta)_{j}$ for all $j \in \mathbb{J}_{k}$. Finally, for each $k \in \mathbb{K}$ and $j \in \mathbb{J}_{k}$, we define the function $\tau_{j}^{(k)}: V \Gamma \rightarrow \mathbb{N}$ by

$$
\tau_{j}^{(k)}(v)=\left|\left\{\Lambda \in \mathscr{L}_{k}: v \in(V \Lambda)_{j}\right\}\right|
$$

For $\Lambda_{0} \in \mathscr{L}(\Gamma)$ and $n \in \mathbb{N}$, we recursively define the subgraphs

$$
\begin{aligned}
\Gamma_{0}\left(\Lambda_{0}\right) & =\Lambda_{0}, \\
\Gamma_{n+1}\left(\Lambda_{0}\right) & =\bigcup\left\{\Lambda \in \mathscr{L}(\Gamma): V \Lambda \cap V \Gamma_{n}\left(\Lambda_{0}\right) \neq \emptyset\right\} .
\end{aligned}
$$

Lemma 2.1 ([5, Lemma 3.1]). Let $\Lambda, \Theta \in \mathscr{L}(\Gamma)$ and let $n \in \mathbb{N}$. If for each $k \in \mathbb{K}$ and $j \in \mathbb{J}_{k}$, the function $\tau_{j}^{(k)}$ is constant on $V \Gamma$, then any isomorphism $\sigma_{n}: \Gamma_{n}(\Lambda) \rightarrow \Gamma_{n}(\Theta)$ admits an extension to an automorphism $\sigma \in \operatorname{Aut}(\Gamma)$.

\footnotetext{
${ }^{2}$ For a short algebraic proof that all 1-ended planar graphs with primitive automorphism group are biconnected, see [8].
} 
This lemma was used in [5] to prove the following characterization of vertex-transitive graphs of connectivity 1 .

Theorem 2.2 ([5, Theorem 3.2]). Let $\Gamma$ be a graph of connectivity 1. A necessary and sufficient condition for $\Gamma$ to be vertex-transitive is that all the functions $\tau_{j}^{(k)}$ be constant on $V \Gamma$.

Notation. When all the lobes of the graph $\Gamma$ are pairwise isomorphic, that is, the index set $\mathbb{K}$ has but one element, then in Equation (2.1) the index $k$ is suppressed; we simply replace $\mathbb{J}_{k}$ by $\mathbb{J}$ and $\tau_{j}^{(k)}$ by $\tau_{j}$.

\section{Lobe-transitivity}

Let $\Gamma$ be a graph of connectivity 1 . It is immediate from the above definitions that the edge-sets of the lobes of $\Gamma$ are blocks of imprimitivity of the group $\operatorname{Aut}(\Gamma)$ acting on $E \Gamma$. Hence any automorphism of $\Gamma$ must map lobes onto lobes, and therefore, if $\operatorname{Aut}(\Gamma)$ is to act transitively on $\mathscr{L}(\Gamma)$, then all the lobes of $\Gamma$ must be pairwise isomorphic. However, pairwise-isomorphism of the lobes alone is not sufficient for lobe-transitivity, even when every vertex of $\Gamma$ lies in the same number of lobes.

Let us first dispense with trees; the proof is elementary and hence omitted.

Proposition 3.1. A finite or countable tree is lobe-transitive (and simultaneously, edgetransitive) if and only if there exist $n_{1}, n_{2} \in \mathbb{N} \cup\left\{\aleph_{0}\right\}$ such that every edge has one incident vertex of valence $n_{1}$ and the other of valence $n_{2}$. If $n_{1}=n_{2}$, the tree is also arc-transitive.

For graphs of connectivity 1 other than trees, we have the following characterization of lobe-transitivity.

Theorem 3.2. Let $\Gamma$ be a graph of connectivity 1 , and let $\Lambda_{0}$ be an arbitrary lobe of $\Gamma$. Let $\left\{P_{i}: i \in \mathbb{I}\right\}$ be the set of orbits of $\operatorname{Aut}(\Gamma)$, and let $\mathscr{Q}=\left\{Q_{j}: j \in \mathbb{J}\right\}$ be the set of those orbits of the stabilizer in $\operatorname{Aut}(\Gamma)$ of $\Lambda_{0}$ that are contained in $\Lambda_{0}$. Then necessary and sufficient conditions for the graph $\Gamma$ to be lobe-transitive are:

(1) For each lobe $\Lambda \in \mathscr{L}(\Gamma)$, there exists an isomorphism $\sigma_{\Lambda}: \Lambda_{0} \rightarrow \Lambda$.

(2) For each $j \in \mathbb{J}$, there exists a function $\tau_{j}: V \Gamma \rightarrow \mathbb{N} \cup\left\{0, \aleph_{0}\right\}$ such that

(a) for all $v \in V \Gamma$,

$$
\tau_{j}(v)=\left|\left\{\Lambda \in \mathscr{L}(\Gamma): v \in \sigma_{\Lambda}\left(Q_{j}\right)\right\}\right|
$$

and

(b) for each $i \in \mathbb{I}, \tau_{j}$ is constant on $P_{i}$ and is nonzero if and only if $Q_{j} \subset P_{i}$.

Proof. (Necessity) Suppose that $\Gamma$ is lobe-transitive. For each lobe $\Lambda \in \mathscr{L}(\Gamma)$, there is an automorphism $\bar{\sigma}_{\Lambda} \in \operatorname{Aut}(\Gamma)$ that maps the fixed lobe $\Lambda_{0}$ onto $\Lambda$. The restriction to $\Lambda_{0}$ of $\bar{\sigma}_{\Lambda}$ is an isomorphism $\sigma_{\Lambda}: \Lambda_{0} \rightarrow \Lambda$ that satisfies condition (1).

For any lobe $\Lambda$, an automorphism $\alpha \in \operatorname{Aut}(\Gamma)$ is in the stabilizer of $\Lambda$ if and only if $\bar{\sigma}_{\Lambda}^{-1} \alpha \bar{\sigma}_{\Lambda}$ is in the stabilizer of $\Lambda_{0}$. It follows that the partition $\left\{\sigma_{\Lambda}\left(Q_{j}\right): j \in \mathbb{J}\right\}$ of $V \Lambda$ is the set of orbits of the stabilizer of $\Lambda$ that are contained in $\Lambda$. Furthermore, since the stabilizer of $\Lambda_{0}$ is a subgroup of $\operatorname{Aut}(\Gamma)$, the partition $\left\{\sigma_{\Lambda}\left(Q_{j}\right): j \in \mathbb{J}\right\}$ of $V \Lambda$ 
refines the partition $\left\{P_{i} \cap V \Lambda: i \in \mathbb{I}\right\}$. If for some indices $i$ and $j$, the vertex $v$ satisfies $v \in \sigma_{\Lambda}\left(Q_{j}\right) \subset P_{i}$, then for any lobe $\Theta$, the vertex $\sigma_{\Theta} \sigma_{\Lambda}^{-1}(v)$ lies in $P_{i} \cap \sigma_{\Theta}\left(Q_{j}\right)$. This implies that, for all $j \in \mathbb{J}$, the function $\tau_{j}$ as given in Equation (3.1) is well-defined and constant on $P_{i}$.

Suppose that for an arbitrary index $i \in \mathbb{I}$, the vertex $v$ lies in $P_{i}$. Since by Equation (3.1), $\tau_{j}(v)$ counts for each $j \in \mathbb{J}$ the number of lobes $\Lambda$ such that $v$ lies in $\sigma_{\Lambda}\left(Q_{j}\right)$, it follows that $\tau_{j}(v)$ is positive exactly when $\sigma_{\Lambda}^{-1}(v) \in Q_{j} \subset P_{i}$ holds, concluding the proof of condition (2.b).

(Sufficiency) Assume conditions (1) and (2). To prove that $\Gamma$ is lobe-transitive, it suffices to prove that every isomorphism $\sigma_{\Theta}: \Lambda_{0} \rightarrow \Theta$ is extendable to an automorphism of $\Gamma$. (Note that in this direction of the proof, $\sigma_{\Theta}$ is not presumed to be the restriction to $\Lambda_{0}$ of an automorphism $\bar{\sigma}_{\Theta} \in \operatorname{Aut}(\Gamma)$ but, in fact, it is.)

Fix a lobe $\Theta_{0}$ and a vertex $v \in V \Lambda_{0}$ and let $w=\sigma_{\Theta_{0}}(v)$. For some $j \in \mathbb{J}$, the vertex $v$ lies in $Q_{j}$, and so $w \in \sigma_{\Theta_{0}}\left(Q_{j}\right)$. Since both $\tau_{j}(v)$ and $\tau_{j}(w)$ are therefore positive, both $v$ and $w$ lie in the same orbit $P_{i}$ of $\operatorname{Aut}(\Gamma)$ by condition (2.b). Furthermore, since $\tau_{j}$ is constant on $P_{i}$ for each $j \in \mathbb{J}$, there exists a bijection $\beta_{j}$ from the set of lobes $\Lambda$ such that $v \in \sigma_{\Lambda}\left(Q_{j}\right)$ onto the set of lobes $\Theta$ such that $w \in \sigma_{\Theta}\left(Q_{j}\right)$. Let $\Lambda_{1}$ be a lobe in the former set, and let $\Theta_{1}=\beta_{j}\left(\Lambda_{1}\right)$.

Although $v$ and $w$ lie in the images of the same orbit $Q_{j}$ in lobes $\Lambda_{1}$ and $\Theta_{1}$, respectively, the vertices $\sigma_{\Lambda_{1}}^{-1}(v)$ and $\sigma_{\Theta_{1}}^{-1}(w)$ need not be the same vertex of $\Lambda_{0}$. However, since both vertices lie in the same orbit $Q_{j}$ of $\Lambda_{0}$, there exists an automorphism $\alpha \in \operatorname{Aut}\left(\Lambda_{0}\right)$ such that $\alpha \sigma_{\Lambda_{1}}^{-1}(v)=\sigma_{\Theta_{1}}^{-1}(w)$. Then $\sigma_{\Theta_{1}} \alpha \sigma_{\Lambda_{1}}^{-1}$ is an isomorphism from $\Lambda_{1}$ onto $\Theta_{1}$ that maps $v$ onto $w$ and therefore agrees with $\sigma_{\Theta_{0}}$ at the vertex $v$ common to $\Lambda_{0}$ and $\Lambda_{1}$.

The amalgamation of $\sigma_{\Theta_{1}} \alpha \sigma_{\Lambda_{1}}^{-1}$ with $\sigma_{\Theta_{0}}$ is an isomorphism from $\Lambda_{0} \cup \Lambda_{1}$ to $\Theta_{0} \cup \Theta_{1}$. By repeating this same technique, we can extend $\sigma_{\Theta_{0}}$ to all lobes adjacent to $\Lambda_{0}$ and then to all of their adjacent lobes and inductively to all of $\Gamma$.

Example 3.3. Suppose that the lobes of $\Gamma$ are copies of some biconnected, vertex-transitive graph and that every vertex of $\Gamma$ is incident with exactly $m$ lobes where $m \geq 2$. By Theorem 2.2, $\Gamma$ is vertex-transitive. By Theorem 3.2, $\Gamma$ is lobe-transitive, with $\mathscr{O}(\operatorname{Aut}(\Gamma))$ being the trivial partition (with just one big cell $P_{1}$ ). Also $|\mathbb{J}|=1$ and $\tau_{1}(v)=m$ for all $v \in V \Gamma$.

Remark 3.4. There exists a "degenerate" family of lobe-transitive graphs $\Gamma$ of connectivity 1 that have but a single cut-vertex. For some cardinal $\mathfrak{K} \geq 2$, consider a collection of $\mathfrak{K}$ copies of a biconnected graph $\Lambda_{0}$, and let $v_{0} \in V \Lambda_{0}$. Let $\sigma_{\Lambda}: \Lambda_{0} \rightarrow \Lambda$ be an isomorphism as in Theorem 3.2, and let $\sigma_{\Lambda}\left(v_{0}\right)=v_{\Lambda}$ for each copy $\Lambda$ of $\Lambda_{0}$ in the collection. We obtain $\Gamma$ by identifying $v_{0}$ and all the vertices $v_{\Lambda}$ and naming the new amalgamated vertex $w$, which forms a singleton orbit $\{w\}$ of $\operatorname{Aut}(\Gamma)$. Clearly $\Gamma$ is lobe-transitive and $w$ is its unique cut-vertex. If $\Lambda_{0}$ has finite diameter, then $\Gamma$ has zero ends (see [3]) when $\Lambda_{0}$ is finite and has $\mathfrak{K}$ ends when $\Lambda_{0}$ is infinite; if $\Lambda_{0}$ has infinite diameter, then $\Gamma$ has at least $\mathfrak{K}$ ends. Other than the graphs just described, all countable lobe-transitive graphs of connectivity 1 are "tree-like" with $\aleph_{0}$ cut vertices and either 2 or $2^{\aleph_{0}}$ ends.

Theorem 3.5. Let $\Lambda_{0}$ be any biconnected graph. Let $H \leq \operatorname{Aut}\left(\Lambda_{0}\right)$, let $\mathscr{Q}=\mathscr{O}(H)=$ $\left\{Q_{j}: j \in \mathbb{J}\right\}$, and let $\mathscr{R}=\left\{R_{k}: k \in \mathbb{K}\right\}$ be a partition of $V \Lambda_{0}$ refined by $\mathscr{Q}$. For each $k \in \mathbb{K}$, let the function $\mu_{k}: \mathbb{J} \rightarrow \mathbb{N} \cup\left\{0, \aleph_{0}\right\}$ satisfy $\mu_{k}(j)>0$ if and only if $Q_{j} \subseteq R_{k}$ and (to avoid the triviality of a single lobe) $\sum_{j \in \mathbb{J}} \mu_{k}(j) \geq 2$ for at least one $k \in \mathbb{K}$. Then there exists (up to isomorphism) a unique lobe-transitive graph $\Gamma$ of connectivity 1 such that 
(1) for each lobe $\Lambda \in \mathscr{L}(\Gamma)$, there exists an isomorphism $\sigma_{\Lambda}: \Lambda_{0} \rightarrow \Lambda$;

(2) for each vertex $v \in V \Gamma$ and each $j \in \mathbb{J}$, we have

$$
\mu_{k}(j)=\left|\left\{\Lambda \in \mathscr{L}(\Gamma): \sigma_{\Lambda}^{-1}(v) \in Q_{j} \subseteq R_{k}\right\}\right| .
$$

Proof. Let $\Lambda_{0}, H, \mathscr{Q}$, and $\mu_{k}$ be as postulated. Let $\Gamma_{0}=\Lambda_{0}$ from which we construct $\Gamma_{1}$ as follows.

Let $v$ be any vertex of $\Lambda_{0}$. For some $j, k$, it must hold that $v \in Q_{j} \subseteq R_{k}$, and so $\mu_{k}(j)>0$. For each $\ell$ such that $Q_{\ell} \subseteq R_{k}$, we postulate the existence of $\mu_{k}(\ell)$ copies $\Lambda$ of $\Lambda_{0}$ (including $\Lambda_{0}$ itself when $\ell=j$ ) such that, if $\sigma_{\Lambda}: \Lambda_{0} \rightarrow \Lambda$ is an isomorphism, then some vertex in $\sigma_{\Lambda}\left(Q_{\ell}\right)$ is identified with the vertex $v$. The graph $\Gamma_{1}$ is produced by repeating this process for each vertex of $\Lambda_{0}$. We repeat this process starting at each vertex $w \in V \Gamma_{1} \backslash V \Gamma_{0}$, the only notational change being that, if specifically $w \in \sigma_{\Lambda}\left(Q_{j^{\prime}}\right)$ for some $j^{\prime} \in \mathbb{J}$, then we consider the subset $\sigma_{\Lambda}\left(Q_{j^{\prime}}\right)$ of $V \Lambda\left(\right.$ instead of $Q_{j}$ in $\left.\Lambda_{0}\right)$ to which $w$ belongs. Thus we construct $\Gamma_{2}$.

Inductively, suppose that $\Gamma_{n}$ has been constructed for some $n \geq 2$. Let $w \in V \Gamma_{n} \backslash$ $V \Gamma_{n-1}$, and so $w \in \sigma_{\Lambda}\left(Q_{m}\right)$ holds for some $m \in \mathbb{J}$ and a unique lobe $\Lambda \in \mathscr{L}\left(\Gamma_{n}\right) \backslash$ $\mathscr{L}\left(\Gamma_{n-1}\right)$. Supposing that $Q_{m} \subseteq R_{k}$, we postulate the existence of $\mu_{k}(m)$ new copies of $\Lambda_{0}$ that share only the vertex $w$ with $\Gamma_{n}$ according to the above identification. In this way we construct $\Gamma_{n+1}$. Finally, let $\Gamma=\bigcup_{n=0}^{\infty} \Gamma_{n}$.

It remains only to prove that $\Gamma$ so-constructed is lobe-transitive. Let $\Theta$ be any lobe of $\Gamma$. By the above construction, all lobes of $\Gamma$ are pairwise isomorphic, and so there exists an isomorphism $\sigma_{\Theta}: \Lambda_{0} \rightarrow \Theta$. Starting with $\Gamma_{0}^{\prime}=\Theta$ and by using the technique in the proof of Sufficiency in Theorem 3.2, one constructs a sequence $\Gamma_{0}^{\prime}, \Gamma_{1}^{\prime}, \ldots$ so that for all $n \in \mathbb{N}$, we have $\Gamma_{n}^{\prime} \cong \Gamma_{n}$, and $\sigma_{\Theta}$ is extendable to an isomorphism from $\Gamma_{n}$ to $\Gamma_{n}^{\prime}$. Thus $\Gamma \cong \bigcup_{n=0}^{\infty} \Gamma_{n}^{\prime}$, and $\sigma_{\Theta}$ can be extended to an automorphism of $\Gamma$.

Example 3.6. In the notation of Theorem 3.5, let $\Lambda_{0}$ be the 5 -cycle with one chord as shown in Figure 1(a), and let $H=\operatorname{Aut}(\Lambda)$, yielding the orbit partition $\left\{Q_{1}, Q_{2}, Q_{3}\right\}$ as indicated. Let $R_{1}=Q_{1} \cup Q_{3}$ and $R_{2}=Q_{2}$, giving $\mathbb{J}=\{1,2,3\}$ and $\mathbb{K}=\{1,2\}$. Define $\mu_{1}(1)=3, \mu_{1}(3)=1$, and $\mu_{2}(2)=2$. Note that all other values of $\mu_{1}$ and $\mu_{2}$ must equal 0 . Then $\Gamma_{1}$ is as seen in Figure 1(b).

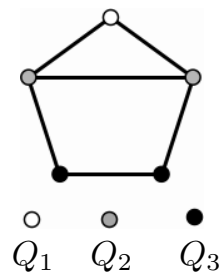

(a) $\Lambda_{0}$

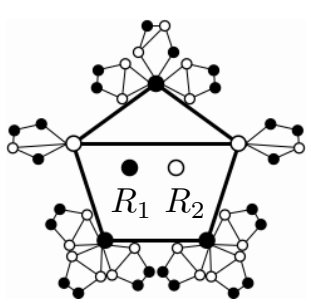

(b) $\Gamma_{1}$

Figure 1: $\Lambda_{0}$ and $\Gamma_{1}$ from Example 3.6.

The pairs of conditions in Theorems 3.2 and 3.5 may appear alike, but there is a notable difference between them. This occurs when the arbitrarily chosen subgroup $H \leq \operatorname{Aut}\left(\Lambda_{0}\right)$ 
of Theorem 3.5 is a proper subgroup of the stabilizer of $\Lambda_{0}$ in $\operatorname{Aut}(\Gamma)$, where $\Gamma$ is the graph constructed from $\Lambda_{0}$ and the functions $\mu_{k}$ of Theorem 3.5. We illustrate this distinction with following example.

Example 3.7. Our initial lobe $\Lambda_{0}$ is a copy of $K_{4}$, with vertices labeled as in Figure 2(a), and so $\operatorname{Aut}\left(\Lambda_{0}\right) \cong \operatorname{Sym}(4)$ of order 24 . We use $\Lambda_{0}$ to "build" the lobe-transitive graph $\Gamma$ shown four times in Figure 2(b). The action on $\Lambda_{0}$ by the stabilizer of $\Lambda_{0}$ in $\operatorname{Aut}(\Gamma)$ is the 4-element group $\left\langle g_{1}\right\rangle \times\left\langle g_{2}\right\rangle$ whose generators have cycle representation $g_{1}=\left(v_{1}, v_{2}\right)$ and $g_{2}=\left(v_{3}, v_{4}\right)$. The shadings of the vertices in the four depictions of $\Gamma$ in Figure 2(b) correspond respectively to the four different subgroups of $\left\langle g_{1}\right\rangle \times\left\langle g_{2}\right\rangle$ described below. For the sake of simplicity, we assume $\mathscr{R}=\mathscr{Q}$.

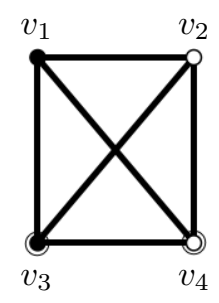

(a)
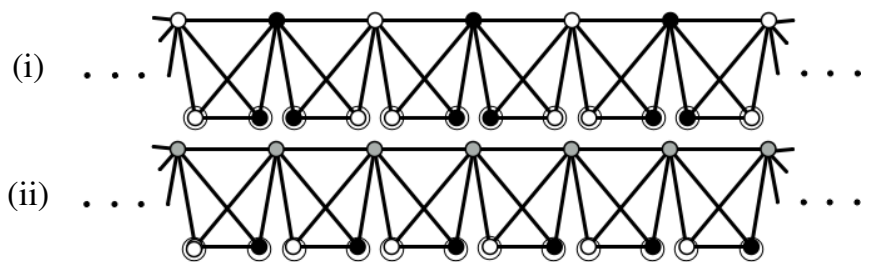

(iii)

(iv)

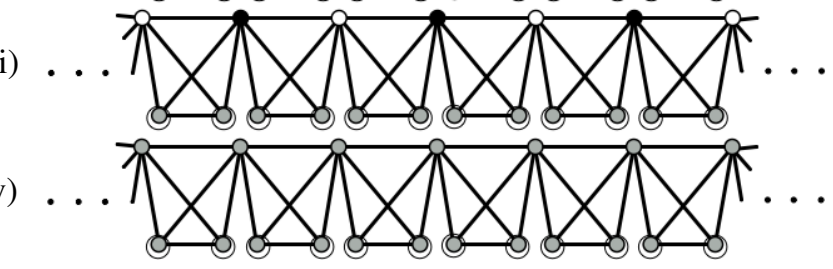

(b)

Figure 2: The clothesline graph.

(i) $H$ is the trivial group $\{\iota\}$. Thus $H$ induces four orbits $Q_{j}=\left\{v_{j}\right\}$ for $j \in\{1,2,3,4\}$. The functions $\mu_{k}$ are then given by $\mu_{k}(j)=2 \delta_{j, k}$ for $k \in\{1,2\}$ and $\mu_{k}(j)=\delta_{j, k}$ for $k \in\{3,4\}$.

(ii) $H=\left\langle g_{1}\right\rangle$. There are three orbits of $H: Q_{1}=\left\{v_{1}, v_{2}\right\}, Q_{2}=\left\{v_{3}\right\}$, and $Q_{3}=\left\{v_{4}\right\}$, which give $\mu_{1}(1)=2, \mu_{2}(2)=\mu_{3}(3)=1$. All other functional values are zero.

(iii) $H=\left\langle g_{2}\right\rangle$. Again there are three orbits of $H$ but not the same ones: $Q_{1}=\left\{v_{1}\right\}$, $Q_{2}=\left\{v_{2}\right\}$, and $Q_{3}=\left\{v_{3}, v_{4}\right\}$. This gives $\mu_{k}(j)=2 \delta_{j, k}$ for $k \in\{1,2\}$ and $\mu_{3}(j)=\delta_{j, 3}$.

(iv) $H=\left\langle g_{1}\right\rangle \times\left\langle g_{2}\right\rangle$. Now there are just two orbits: $Q_{1}=\left\{v_{1}, v_{2}\right\}$ and $Q_{2}=\left\{v_{3}, v_{4}\right\}$. Finally, $\mu_{1}(1)=2, \mu_{2}(2)=1$, and all other functional values are zero.

All four choices for $H$, the partition $\mathscr{Q}$, and the functions $\mu_{k}$ clearly yield the same lobetransitive graph $\Gamma$ of connectivity 1 by the construction of Theorem 3.5

\section{Edge-transitivity}

Lemma 4.1. If $\Gamma$ is an edge-transitive (respectively, arc-transitive) graph, then $\Gamma$ is lobetransitive and its lobes are also edge-transitive (respectively, arc-transitive). 
Proof. For $i=1,2$, let $\Theta_{i} \in \mathscr{L}(\Gamma)$, and let $e_{i}$ be an edge (respectively, arc) of $\Theta_{i}$. There exists an an automorphism $\varphi \in \operatorname{Aut}(\Gamma)$ such that $\varphi\left(e_{1}\right)=e_{2}$. Since $\varphi$ maps cycles through $e_{1}$ onto cycles through $e_{2}, \varphi$ must map $\Theta_{1}$ onto $\Theta_{2}$. If $e_{1}$ and $e_{2}$ lie in the same lobe $\Theta$, then $\varphi$ leaves $\Theta$ invariant, and so its restriction to $\Theta$ is an automorphism of $\Theta$.

Theorem 4.2. Let $\Gamma$ be a graph of connectivity 1 with more than one lobe, and let $\Lambda \in$ $\mathscr{L}(\Gamma)$. Necessary and sufficient conditions for $\Gamma$ to be edge-transitive are the following:

(1) The lobes of $\Gamma$ are edge-transitive.

(2) For each lobe $\Theta \in \mathscr{L}(\Gamma)$, there exists an isomorphism $\sigma_{\Theta}: \Lambda \rightarrow \Theta$.

(3) Exactly one of the following descriptions of $\Gamma$ holds:

(a) Both $\Gamma$ and $\Lambda$ are vertex-transitive, in which case every vertex is incident with the same number $\geq 2$ of lobes.

(b) The graph $\Gamma$ is vertex-transitive but $\Lambda$ is not vertex-transitive, in which case $\Lambda$ is bipartite with bipartition $\left\{Q_{1}, Q_{2}\right\}$, and there exist constants $m_{1}, m_{2} \in$ $\mathbb{N} \cup\left\{\aleph_{0}\right\}$ such that for $j=1,2$ and all $v \in V \Gamma$, it holds that

$$
m_{j}=\left|\left\{\Theta \in \mathscr{L}(\Gamma): v \in \sigma_{\Theta}\left(Q_{j}\right)\right\}\right| .
$$

(c) The graph $\Gamma$ is not vertex-transitive, in which case $\Gamma$ is bipartite with bipartition $\left\{P_{1}, P_{2}\right\}$ and there exist constants $m_{1}, m_{2} \in \mathbb{N} \cup\left\{\aleph_{0}\right\}$, at least one of which is at least 2 , such that for $i=1,2$, if $v \in P_{i}$, then

$$
\left|\left\{\Theta \in \mathscr{L}(\Gamma): v \in \sigma_{\Theta}\left(P_{j} \cap V \Lambda\right)\right\}\right|=m_{j} \delta_{i, j}
$$

Proof. (Sufficiency) Assume all the conditions in the hypothesis and let $e_{1}, e_{2} \in E \Gamma$ be arbitrary edges in lobes $\Lambda_{1}$ and $\Lambda_{2}$, respectively. By condition (2), there exists an isomorphism $\sigma: \Lambda_{1} \rightarrow \Lambda_{2}$. By condition (1), there exists an automorphism $\alpha \in \operatorname{Aut}\left(\Lambda_{2}\right)$ such that $e_{2}=\alpha \sigma\left(e_{1}\right)$. Each of the three cases of condition (3) is seen to satisfy the hypothesis of Lemma 2.1, implying that $\alpha \sigma: \Lambda_{1} \rightarrow \Lambda_{2}$ is extendable to an isomorphism of $\Gamma$ mapping $e_{1}$ to $e_{2}$.

(Necessity) Suppose that $\Gamma$ is an edge-transitive graph of connectivity 1 . By Lemma 4.1, $\Gamma$ is also lobe-transitive and its lobes are edge-transitive, proving condition (1). Condition (2), which establishes notation for the remainder of this proof, also follows from Lemma 4.1.

To prove (3), we continue the notation of Theorem 3.2 with $\mathbb{I}$ being the index set for the set of orbits of $\operatorname{Aut}(\Gamma)$ and $\mathbb{J}$ being the index set for the orbits of the stabilizer of $\Lambda$ that are contained in $\Lambda$. Since both $\Gamma$ and all of its lobes are edge-transitive, $|\mathbb{I}|$ and $|\mathbb{J}|$ equal either 1 or 2 .

If both $\Gamma$ and $\Lambda$ are vertex-transitive, then $|\mathbb{I}|=|\mathbb{J}|=1$, and for every vertex $v \in V \Gamma$, $\tau_{1}(v)=m$ holds for some $m \geq 2$. This is case (3.a).

Since any odd cycle in $\Gamma$ would be contained in a lobe of $\Gamma$, it holds that $\Gamma$ is bipartite if and only if every lobe is bipartite. If either $\Gamma$ or $\Lambda$ is not vertex-transitive, then each and hence both - are bipartite, and the sides of the bipartitions (whether or not they are entire orbits of the appropriate automorphism group) are blocks of imprimitivity systems. Let $\left\{P_{1}, P_{2}\right\}$ be the bipartition of $V \Gamma$, and so $\left\{P_{1} \cap V \Theta, P_{2} \cap V \Theta\right\}$ is the bipartition of 
any lobe $\Theta$. Equivalently, letting $\left\{Q_{1}, Q_{2}\right\}$ denote the bipartition of $\Lambda$, we have $P_{i}=$ $\bigcup_{\Theta \in \mathscr{L}(\Gamma)} \sigma_{\Theta}\left(Q_{i}\right)$ for $i=1,2$.

Suppose that $\Gamma$ is vertex-transitive but $\Lambda$ is not, and so $|\mathbb{I}|=1$ and $|\mathbb{J}|=2$. By Theorem 2.2, there exist constants $m_{1}, m_{2} \in \mathbb{N} \cup\left\{\aleph_{0}\right\}$ such that for all $v \in V \Gamma$ and $j \in \mathbb{J}$, we have $m_{j}=\tau_{j}(v)=\left|\left\{\Theta \in \mathscr{L}(\Gamma): v \in \sigma_{\Theta}\left(Q_{j}\right)\right\}\right|$.

Finally, suppose that $\Gamma$ is not vertex-transitive, and so $P_{1}$ and $P_{2}$ are the orbits of $\operatorname{Aut}(\Gamma)$. Also, $\Lambda$ is bipartite with bipartition $\left\{Q_{1}, Q_{2}\right\}$, where $Q_{i}=P_{i} \cap V \Lambda$. As no automorphism of $\Gamma$ swaps $P_{1}$ with $P_{2}$, no automorphism of $\Gamma$ swaps $Q_{1}$ with $Q_{2}$ (even though $\Lambda$ may be vertex-transitive!). Hence $|\mathbb{I}|=|\mathbb{J}|=2$. Since $\Gamma$ is lobe-transitive, it follows now from the "necessity" argument of Theorem 3.2 that, for $j=1,2$, the function $\tau_{j}$ satisfies the condition $\tau_{j}(v)>0$ if and only if $v \in P_{j}$. That means that there exist constants $m_{1}, m_{2} \in \mathbb{N} \cup\left\{\aleph_{0}\right\}$, at least one of which is greater than 1 , such that, if $v \in P_{i}$, then $\tau_{j}(v)=m_{j} \delta_{i, j}$.

Example 4.3. Suppose in the notation of Theorem 4.2 that $\Gamma$ is edge-transitive and $\Lambda$ is the complete bipartite graph $K_{s, t}$ with $\left|Q_{1}\right|=s$ and $\left|Q_{2}\right|=t$. Suppose that every vertex of $\Gamma$ is incident with exactly two lobes isomorphic to $\Lambda$. If $s=t$, then both $\Gamma$ and $\Lambda$ are vertex-transitive, and we have case (3.a) of the theorem. If $s \neq t$ and every vertex lies in one image of $Q_{1}$ and one image of $Q_{2}$, then we have the situation of case (3.b). If again $s \neq t$ but each vertex lies in either two images of $Q_{1}$ or two images of $Q_{2}$, then we have the situation described in case (3.c).

Remark 4.4. With regard to Example 4.3, we note that having $s=t$ does not assure vertex-transitivity of edge-transitive bipartite graphs. There exist edge-transitive, nonvertex-transitive, finite bipartite graphs where the two sides of the bipartition have the same size. Such graphs are called semisymmetric. The smallest such graph, on 20 vertices with valence 4, was found by J. Folkman [2], who also found several infinite families of semisymmetric graphs. Many more such families as well as forbidden values for $s$ were determined by A. V. Ivanov [4].

Example 4.5. This simple example illustrates how the converse of Lemma 4.1 is false, even though the lobes themselves may be highly symmetric. Let $\Gamma$ be a graph of connectivity 1 whose lobes are copies of the Petersen graph (which is 3-arc-transitive!). For each lobe $\Lambda$, let $(V \Lambda)_{1}$ and $(V \Lambda)_{2}$ denote the vertex sets of disjoint 5-cycles indexed "consistently," i.e., if $\Lambda$ and $\Theta$ share a vertex $v$, then $v \in(V \Lambda)_{i} \cap(V \Theta)_{i}$ for $i=1$ or $i=2$. (Observe that $\Gamma$ is not bipartite.) For $i=1,2$ define $P_{i}=\bigcup_{\Theta \in \mathscr{L}(\Gamma)}(V \Theta)_{i}$, and suppose that each vertex in $P_{i}$ belongs to exactly $m_{i}$ lobes. The graph $\Gamma$ is lobe-transitive by Theorem 3.2 , and $\Gamma$ is both vertex- and edge-transitive when $m_{1}=m_{2}$, but $\Gamma$ is neither vertex- nor edge-transitive when $m_{1} \neq m_{2}$.

\section{Arc-transitivity}

Theorem 5.1. Let $\Gamma$ be a graph of connectivity 1 . Necessary and sufficient conditions for $\Gamma$ to be arc-transitive are the following:

(1) The lobes of $\Gamma$ are arc-transitive.

(2) The lobes of $\Gamma$ are pairwise isomorphic.

(3) All vertices of $\Gamma$ are incident with the same number of lobes. 
Proof. (Necessity) Suppose that $\Gamma$ is arc-transitive. Conditions (1) and (2) follow from Lemma 4.1. Since arc-transitivity implies vertex-transitivity, condition (3) holds.

(Sufficiency) Assume that the three conditions hold. For $k=1,2$, let $a_{k}$ be an arc of $\Gamma$, and let $\Theta_{k}$ be the lobe containing $a_{k}$. By condition (2), there exists an isomorphism $\sigma: \Theta_{1} \rightarrow \Theta_{2}$. By condition (1), there exists an automorphism $\alpha \in \operatorname{Aut}\left(\Theta_{2}\right)$ such that $\alpha \sigma\left(a_{1}\right)=a_{2}$. By condition (3), the functions $\tau_{j}$ of Equation (2.1) are constant on $V \Gamma$. (In fact, since the lobes are vertex-transitive, there is only one such function.) It now follows from Lemma 2.1 that $\alpha \sigma$ is extendable to all of $\Gamma$.

Remark 5.2. If conditions (1) and (3) of Theorem 5.1 were replaced by the lobes are edgetransitive and $\Gamma$ is vertex-transitive, the sufficiency argument would fail. There exist finite graphs [1] and countably infinite graphs with polynomial growth rate [7] that are vertexand edge-transitive but not arc-transitive. Let $\Lambda$ denote such a graph, and consider a graph $\Gamma$ whose lobes are isomorphic to $\Lambda$ with the same number of lobes incident with every vertex. Then $\Gamma$ itself is vertex- and edge-transitive but not arc-transitive.

The following proposition is elementary.

Proposition 5.3. For all $k \geq 2$, the only $k$-arc-transitive graphs of connectivity 1 are trees of constant valence.

\section{References}

[1] I. Z. Bouwer, Vertex and edge transitive, but not 1-transitive, graphs, Canad. Math. Bull. 13 (1970), 231-237, doi:10.4153/cmb-1970-047-8.

[2] J. Folkman, Regular line-symmetric graphs, J. Comb. Theory 3 (1967), 215-232, doi:10.1016/ s0021-9800(67)80069-3.

[3] R. Halin, Automorphisms and endomorphisms of infinite locally finite graphs, Abh. Math. Sem. Univ. Hamburg 39 (1973), 251-283, doi:10.1007/bf02992834.

[4] A. V. Ivanov, On edge but not vertex transitive regular graphs, in: C. J. Colbourn and R. Mathon (eds.), Combinatorial Design Theory, North-Holland, Amsterdam, volume 149 of NorthHolland Mathematics Studies, pp. 273-285, 1987, doi:10.1016/s0304-0208(08)72893-7.

[5] H. A. Jung and M. E. Watkins, On the structure of infinite vertex-transitive graphs, Discrete Math. 18 (1977), 45-53, doi:10.1016/0012-365x(77)90005-x.

[6] O. Ore, Theory of Graphs, volume XXXVIII of American Mathematical Society Colloquium Publications, American Mathematical Society, Providence, Rhode Island, 1962.

[7] C. Thomassen and M. E. Watkins, Infinite vertex-transitive, edge-transitive non-1-transitive graphs, Proc. Amer. Math. Soc. 105 (1989), 258-261, doi:10.2307/2046766.

[8] J. Širáň and M. E. Watkins, Imprimitivity of locally finite, 1-ended, planar graphs, Ars Math. Contemp. 5 (2012), 213-217, doi:10.26493/1855-3974.213.fcd.

[9] M. E. Watkins, Les graphes de Cayley de connectivité un, in: Problèmes combinatoires et théorie des graphes, CNRS, Paris, volume 260 of Colloques Internationaux du Centre National de la Recherche Scientifique, pp. 419-422, 1978, Colloque International CNRS held at the Université d'Orsay, Orsay, July 9 - 13, 1976.

[10] M. E. Watkins, Infinite graphical Frobenius representations, Electron. J. Combin. 25 (2018), \#P4.22 (23 pages), https://www.combinatorics.org/ojs/index.php/eljc/ article/view/v25i4p22.

[11] M. E. Watkins and J. E. Graver, A characterization of infinite planar primitive graphs, J. Comb. Theory Ser. B 91 (2004), 87-104, doi:10.1016/j.jctb.2003.10.005. 